\title{
Current Enrollment
}

National Cancer Institute

\section{Source}

National Cancer Institute. Current Enrollment. NCI Thesaurus. Code C142467.

The number of active participants. 